\title{
Computations of Coupled Electronic States in Quantum Dot/Wetting Layer Cylindrical Structures
}

\author{
Roderick V.N. Melnik ${ }^{1}$ and Kate N. Zotsenko ${ }^{2}$ \\ 1 University of Southern Denmark, \\ Mads Clausen Institute, DK-6400, Denmark, rmelnik@mci.sdu.dk \\ 2 Electronic Data Systems Australia, \\ 60 Currington Street, Sydney, NSW 2000, Australia
}

\begin{abstract}
There exist several techniques allowing to confine the motion of an electron from all three dimensions. The resulting structures, known as quantum dots, promise new opportunities in optoelectronics, and a better understanding of such structures requires incorporating new effects into existing models which inevitably leads to the necessity of using efficient computational tools. In this paper we analyse computationally the electronic states in quantum dots of cylindrical shape taking into account the influence of the wetting layer. Coupling effects between quantum dot and wetting layer electronic states are demonstrated with numerical examples.
\end{abstract}

\section{Introduction}

Microelectronics has provided some of the most challenging problems in computational science [5]. Moving to nanosize devices opens new opportunities for computational scientists, and many important problems in nanoscience have been already attacked with computational tools. The major focus of the present paper is on modelling low-dimensional semiconductor nanostructures. At present, there exist several methodologies allowing to produce electronic states confined from all three dimensions. The resulting structures are known as quantum dots, and one of the most popular techniques of their growth is the Stranski-Krastanow methodology. This methodology is essentially a self-organised hetero-epitaxial growth during molecular beam epitaxy. The properties of the structure resulting from this growth process are dependent on thermal, elastic, piezoelectric, and other effects, and controlling the process is a difficult task [10] which can be assisted by computational experiments. The structures themselves can be grown in different shapes such as pyramidal, ellipsoidal, conical, cylindrical. The geometry of the structure influences substantially optoelectronic properties of the quantum device, as do different effective masses in the dot and the crystal matrix of such devices [4,9]. Recently, it was shown that the inclusion of the wetting layer, a "substrate" on which the dot is grown, is also essential since the wetting layer electronic states and the states of the quantum dot are essentially 
coupled [6]. It is our purpose in this paper to examine further this influence, in particular for cylindrical quantum dots. Although cylindrically-shaped quantum dots have been analysed before (e.g., [8] and references therein), no prior systematic investigation of the influence of the wetting layer on electronic states of the whole structure have been carried out. A better understanding of this influence is important in evaluating optical properties of low-dimensional nanostructures (e.g., [1]).

\section{Position-Dependent Effective Mass Model for Quantum Dot/Wetting Layer Nanostructures}

In this paper we are considering InAs quantum dots embedded in GaAs matrices. We consider the full 3D model of the quantum dot by using the envelope function approximation. The discussion is focused on the one-band model as an example, in which case the Hamiltonian of the system can be represented in the following form (e.g., [7]):

$$
H=-\frac{\hbar^{2}}{2} \nabla_{\mathbf{r}} \cdot\left(\frac{1}{m(\mathbf{r})} \nabla_{\mathbf{r}}\right)+V(\mathbf{r})
$$

where $m(\mathbf{r})$ is the position-dependent electron effective mass and $V(\mathbf{r})$ is the confinement (band-edge) potential. Although it is often assumed that the electron effective mass is a constant inside of the domain of interest, this assumption cannot be justified for any real considerations since $m(\mathbf{r})$ is a function of position and varies considerably between two major regions of interest, InAs and GaAs in this paper. Taking into account this difference is essential, but this is connected with the necessity to interpret the model

$$
H \psi(\mathbf{r})=E \psi(\mathbf{r})
$$

in a variational sense [3] with $E$ being the electron potential energy which takes discrete levels. The quantum dot is grown as a few monolayer InAs structure of cylindrical shape. The structure rests on a thin InAs wetting layer, as it is depicted in Fig. 1. The whole dot/wetting layer InAs structure is embedded in a GaAs crystal matrix. For such geometries the resulting model is not amenable to analytical treatments which have been traditionally applied in the physical literature in this context under much simplified assumptions. Note also that taking into account additional effects, such as elastic, thermal, and piezoelectric to name just a few, would necessarily lead to an increasing attention to the development of efficient computational tools for the models discussed in this paper.

The growth direction, considered in this paper as an example, is (100), in which case the model (1), (2) allows a separation of variables, and can be reduced to

$$
\bar{H} \bar{\psi}(r, z)=E \bar{\psi}(r, z), \quad \psi(\mathbf{r})=\bar{\psi}(r, z) \bar{\Phi}(\phi),
$$


where $\mathrm{n}$ is an integer making function $\bar{\Phi}=\exp (\operatorname{in} \phi)$ a single-valued function such that $\bar{\Phi}(0)=\bar{\Phi}(2 \pi)$, and the Hamiltonian of the problem can be represented in the form

$$
\bar{H}=-\frac{\hbar^{2}}{2}\left[\frac{\partial}{\partial r}\left(\frac{1}{m(r, z)} \frac{\partial}{\partial r}\right)+\frac{\partial}{\partial z}\left(\frac{1}{m(r, z)} \frac{\partial}{\partial z}\right)\right]+\frac{\hbar^{2} n^{2}}{2 m(r, z) r^{2}}+V(r, z) .
$$

The boundary conditions can be derived based on the Ben-Daniel-Duke formulations (e.g., $[2,8]$ ), relating the values of the wave function in the dot material and in the crystal matrix. In this paper we use the idea analogous to that proposed originally in [6].

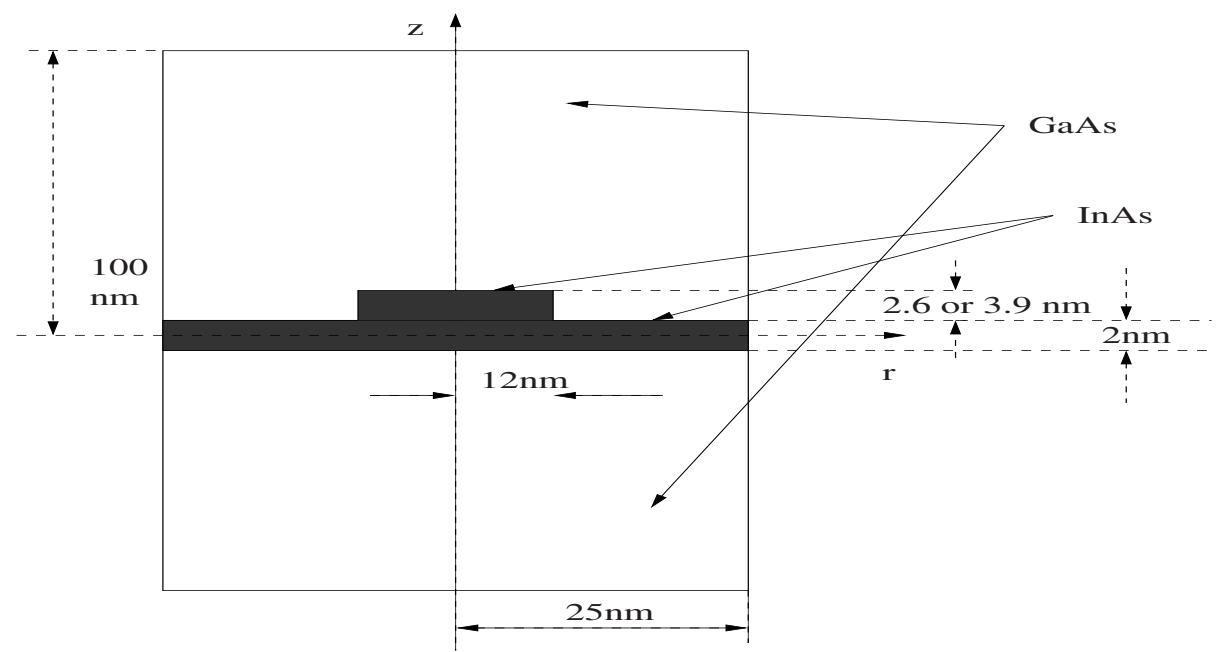

Fig. 1. Quantum dot geometries

\section{Numerical Procedure}

The model formulated in Section 2 is a partial differential equation eigenvalue problem which needs to be solved in order to determine energy levels and the corresponding wave functions of this coupled quantum dot/wetting layer system. The procedure applied in this paper is based on the spectral transformation Arnoldi iterations. This is a Krylov-subspace-based technique which allows us to deal effectively with large matrices resulting from the numerical discretisation of $(3),(4)$. In particular, in solving the discretised problem

$$
[A-((E-V) I] \mathbf{x}=\mathbf{g}
$$

with given matrix $A: n \times n$ and $\mathbf{g}: 1 \times n$ (resulting from the discretisation of (3) and (4)), and with n varying from $10^{4}$ to $10^{5}$, we construct iterations with respect to a sequence of Krylov subspaces

$$
\mathbf{x}, A \mathbf{x}, A^{2} \mathbf{x}, \ldots, A^{m-1} \mathbf{x}, \ldots
$$


Computations have been carried out on a sequence of grids for all numerical results reported in the next section.

Geometric characteristics of the structure under consideration are given in Fig.1. Other parameters used in computations include the conduction-band effective masses $\left(0.023 m^{*}\right.$ and $0.067 m^{*}$ for InAs and GaAs, respectively, with $m^{*}$ being the free electron mass) and band-edge energies (-0.697 and 0 for InAs and GaAs, respectively).

\section{Computational Analysis of Electronic States in QD/WL Coupled Systems}

We present in this paper four groups of experiments. Firstly, we consider a cylindrically shaped quantum dot with wetting layer. The size of the dot is $24 \mathrm{~nm}$ at the basis (the computational domain is half of this value due to the cylindrical symmetry). The height of the cylinder is $2.6 \mathrm{~nm}$. In Fig. 2 we present the ground state and the first three excited states. Recall that for the conical quantum dots with the same base and height the resulting eigenvalues reported in $[6](-0.409,-0.307,-0.253,-0.150)$ are higher compared to their counterparts in the cylindrical case. In the case of cylindrical dots of the specified dimensions they are $-0.474,-0.324,-0.289,-0.170$, respectively (we rounded off all results to include the third digit after the dot only). In both cases, however, the ground state is clearly localisable.

In the second group of experiments we neglect the wetting layer and consider the "pure" quantum dot. In this case, the ground state (see Fig. 3 left) is -0.343 which is substantially larger compared to the result obtained for the quantum dot with wetting layer. The first (the only excited state in this case) is -0.186 , and this state much better confined compared to the corresponding state in the quantum dot/wetting layer case. The wave functions of these two states are represented in Fig. 3.

Next, we consider the wetting layer only. In this case, the profile of the wave function has a zero-slope shape, typical for quantum wells (see Fig. 4 left), corresponding to the ground state eigenvalue which has the value of -0.310 . In this case, the three excited states are $-0.281,-0.212$, and -0.110 , respectively.

By increasing the height of the dot by factor of 1.5 and keeping its width the same, for the dot without wetting layer we obtain values of -0.437 and -0 . 264 for the ground and the first three excited states, respectively, which are the only eigenvalues in this case. As expected, these values are smaller compared to the "pure" quantum dot of smaller height. The ground state wave function in this case is represented in Fig. 4 (right). Finally, we note that the ground state and the first three excited states for the new quantum dot/wetting layer structure correspond to the values of $-0.348,-0.300,-0.182,-0.062$, respectively. These values produce wave functions similar to those presented in Fig. 2, but, as it is expected, their values are larger compared to smaller height cylindrical quantum dots with wetting layers. 

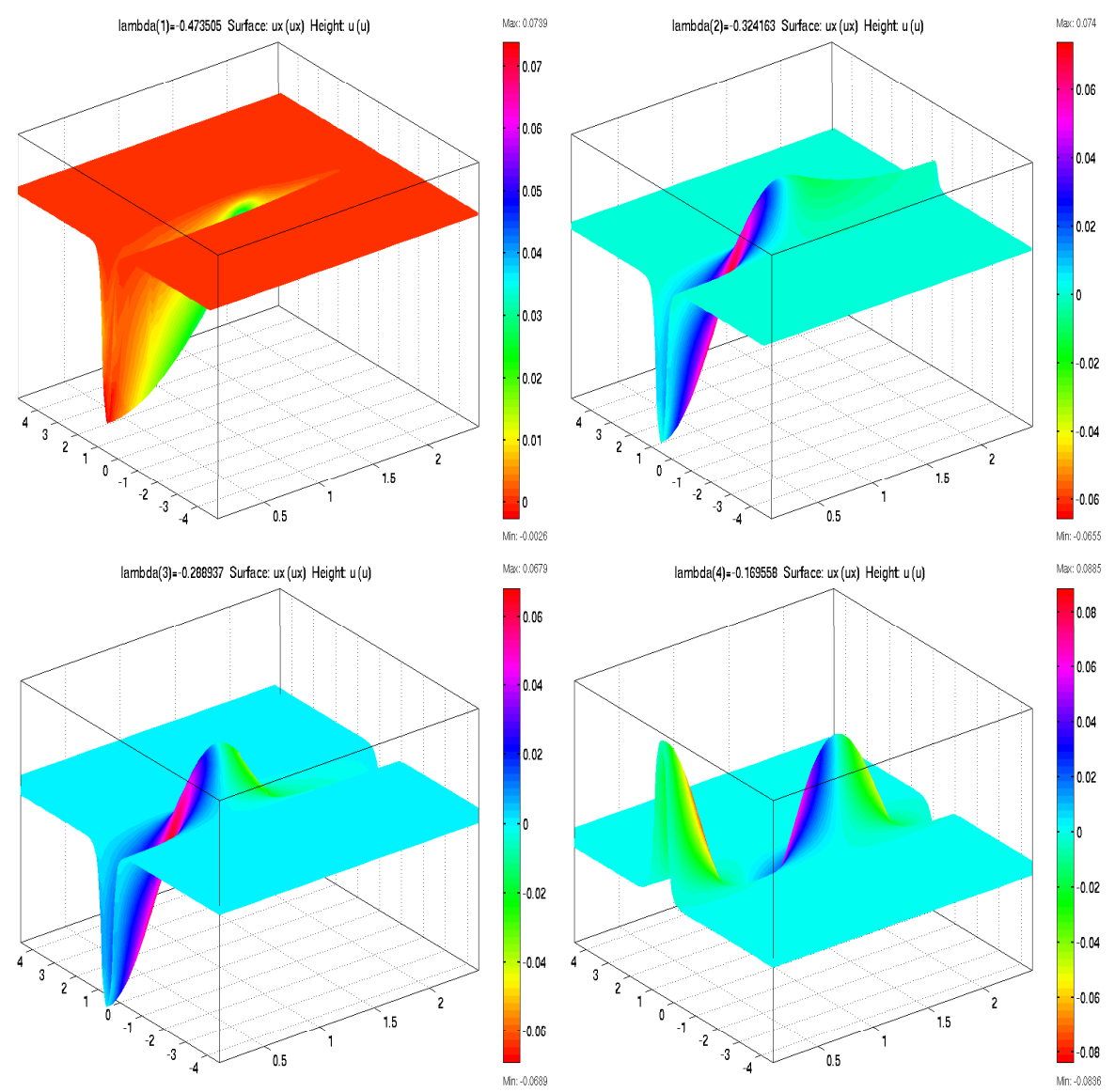

Fig. 2. Cylindrical QD with wetting layer: ground state and the first three excited states.

\section{Acknowledgment.}

The first author is grateful to Morten Willatzen for many fruitful discussions on the topics of this paper.

\section{References}

1. Adeler, F. et al: Optical Transitions and Carrier Relaxation in Self-Assembled InAs/GaAs Quantum Dots. J. Appl. Phys. 80 (1996) 4019-4026

2. Bastard, G.: Wave Mechanics Applied to Semiconductor Heterostructures. Halsted Pressm (1988) 

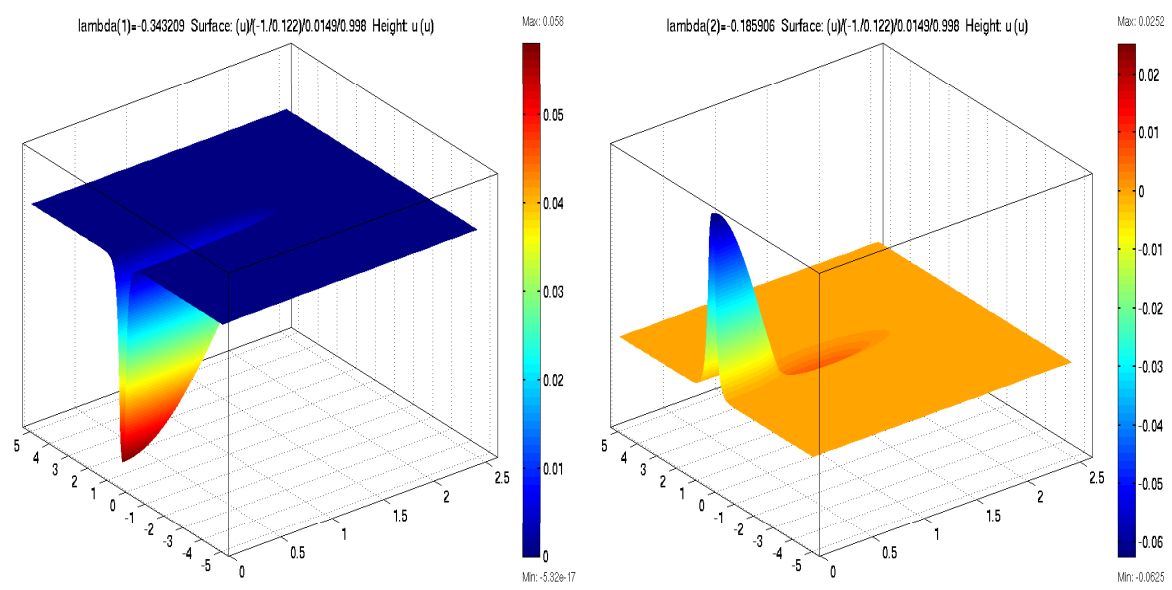

Fig. 3. Cylindrical QD without wetting layer: ground state and the first excited state.
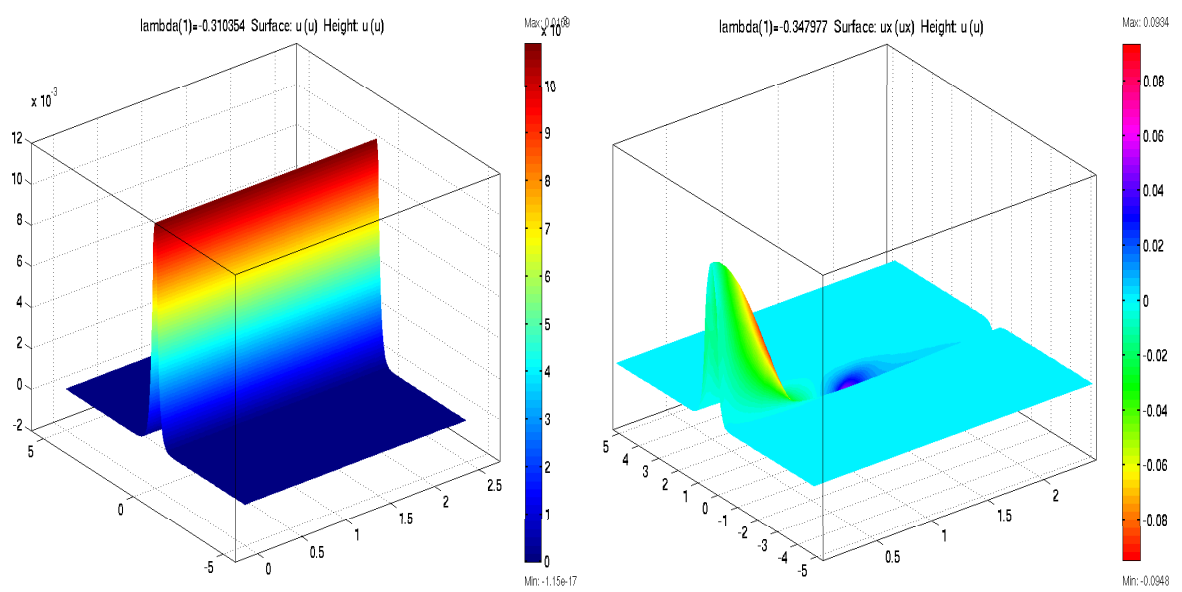

Fig. 4. Ground state of the wetting layer (left) and the ground state of the increasedheight dot with wetting layer (right). 
3. Gelbard, F. and Malloy, K.J.: Modeling Quantum Structures with BEM. J. Comp. Phys. 172 (2001) 19-39

4. Bimberg, D., Grundmann, M., and Ledentsov, N.N.: Quantum Dot Heterostructures. John Wiley and Sons, UK (1998)

5. Melnik, R.V.N., He, H.: Modelling nonlocal processes in semiconductor devices with exponential difference schemes. Journal of Engineering Mathematics. 38 (2000) 233263

6. Melnik, R.V.N. and Willatzen, M.: Modelling Coupled Motion of Electrons in Quantum Dots with Wetting Layers. Proceedings of the Modeling and Simulation of Microsystems (MSM) Conference, April 21-25, 2002, USA. (2002) 506-509

7. Li, Y. et al: Electron Energy Level Calculations for Cylindrical Narrow Gap Semiconductor Quantum Dot. Computer Physics Communications. 140 (2001) 399-404

8. Li, Y. et al: Computer Simulation of Electron Energy Levels for Different Shape InAs/GaAs Semiconductor Quantum Dots. Computer Physics Communications. 141 (2001) 66-72

9. Stier, O., Grundmann, M., and Bimberg, D.: Electronic and Optical Properties of Strained QDs modeled by 8-band $k \cdot p$ Theory. Phys. Rev. B. 59 (1999) 5688-5701

10. Williams, R.L. et al: Controlling the Self-Assembly of InAs/InP Quantum Dots. J. of Crystal Growth. 223 (2001) 321-331 